\title{
Research of a 5GHz Mining Access Point
}

\author{
Xiao Lv ${ }^{1, a^{*}}$, Da Zhang, Feng Jin, Xu Liu, Xuesong Chen \\ ${ }^{1}$ Beijing General Research Institute of Mining \& Metallurgy, Beijing, China \\ agracepenguin@126.com
}

Keywords: $5 G$, WLAN, access point, signal interference.

Abstract. Most existing mining wireless systems are working at $2.4 \mathrm{GHz}$, such as $2.4 \mathrm{GHz} \mathrm{WiFi}$ commutation assess point, Zigbee wireless positioning assess point, various $2.4 \mathrm{GHz}$ wireless communication terminals, $2.4 \mathrm{GHz}$ wireless sensor terminals, etc.. Since the span of $2.4 \mathrm{GHz}$ free frequency is limited, the signal collisions can't be avoided, which would cause packet loss, transmitting delay and other unpredictable issues, then stability of the mining wireless control systems, information acquisition systems, and communication systems are influenced. $5 \mathrm{GHz}$ band is open and free, few mining wireless terminals are working at this band, communicating at $5 \mathrm{GHz}$ can effectively avoid the interference between the wireless signals. Based on this, this paper designed a $5 \mathrm{G}$ mining access point, which solve the $2.4 \mathrm{GHz}$ signal interference problem, ensure the quality of the communication of wireless communication system.

\section{Introduction}

With the development of information technology, kinds of wireless assess points and terminals are using in mining environment, such as mining wireless control systems, wireless information acquisition systems, wireless communication systems and etc. Most of them are working at open free frequency $2.4 \mathrm{GHz}$. Since the span of $2.4 \mathrm{GHz}$ free frequency is limited, the signal collisions can't be avoided, which would cause packet loss, packet delay and other unpredictable issues. These issues will greatly affect the performance of the various systems, such as communication quality, load capacity, system stability.

\section{Related work}

IEEE $802.11 \mathrm{n}$ is the highest rate communication standard [4] which has been large-scale commercial using. Compared to traditional IEEE 802.11 technology, IEEE 802.11n has the following characteristics: 1) MIMO technology; 2) channel bonding, support 20/40 MHz channel; 3) MAC layer frame aggregation. Paper[5] design a 802.11n mining access point which works at $2.4 \mathrm{GHz}$, but due to the signal interference between the $2.4 \mathrm{GHz}$ wireless equipments, the system's throughput, and the system packet loss ratio in open mining environment is influenced. To slove above problem, and to get the similar communication distance, this paper designs and implements a $5 \mathrm{GHz}$ mining high power access point.

\section{G Access Point Design}

This paper designs four major parts in 5GHz Access Point Design: core driver block, 5G radio block design, memory block, power and interface block, shown in Figure 1. Core driver block loads the hardware driver and boot the board, controls the RF chips to work at transmitting or receiving mode. 5G radio block amplifies RF signal baseband chip's output signal, transmit to the antenna, 
filter and amplify the weak radio signal received from the antenna, and transmit to the baseband chip. Power and interface modules provide the necessary working power and peripheral interface.

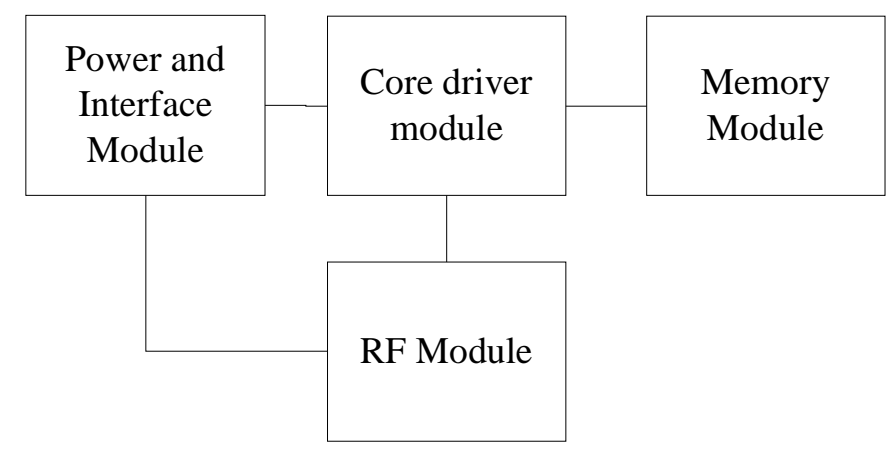

Fig. 1. System block diagram

Core driver module design. The Core driver module is driven by two AR7240 and AR9280 chip. AR7240 is a high performance network processor; it includes a MIPS 24K processor, PCIE host interface, $64 \mathrm{~K}$ instruction cache and $32 \mathrm{~K}$ data cache. AR9280 is a highly integrated single-chip solution for $2.4 \mathrm{G}$ and $5 \mathrm{GHz} 802.11$ n-ready wireless local area networks that enable high-performance $2 \times 2$ MIMO configurations. It integrates a multi-protocol MAC, baseband processor, analog-to-digital and digital-to analog converters, $2 \times 2$ MIMO radio transceiver, and PCI Express interface in an all-CMOS device.

Memory design. Since the core driver module needs to be boot from external flash, paper designs a serial flash and DDR SDRAM to run the code. W25X32VSFIG is 32Mb serial flash that to store the code, including the hardware driver and the software. HY5D4281622FTP is a 128M DDR SRAM; processor runs the code in it.

5G Radio block Design. 5G radio block have several main parts: signal attenuation circuit, filter circuit, output signal amplifying circuit, small input signal amplifying circuit, transmitting/receiving control circuit, power detector circuit. The block diagram is shown in Fig. 2.

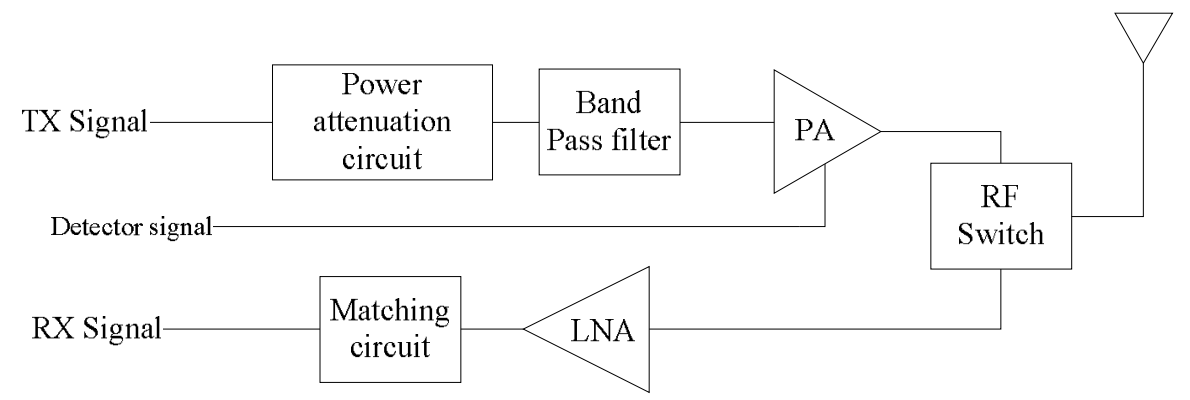

Fig. 2. RF design block diagram

SE5004 is selected in output signal amplification block. SE5004 is a 5G power amplifier whose maximum output power can be up to $26 \mathrm{DBM}$ and the gain is up to $32 \mathrm{db}$. it builts in level 3 amplifying circuit, and the working voltage is $5 \mathrm{v}$. This paper designs the COMA signal from the baseband chip to control the switch of PA. when the signal is not transmitting, the control signal closes the power amplifier, and make the board work in power saving mode, then reduce the power 
consumption of the whole system. When using SE5004, main attention needs to be pay to its power supply, grounding, and $\mathrm{rf} 50 \mathrm{ohm}$ impedance matching. Paper also designs the detect circuit by using the power amplifier. The detector of SE5004 has a analog to digital conversion circuit in it, it can collects the RF signal in the air, converts the energy to digital signal, then gives feedback to the baseband chip. Feedback circuit can be divided into two kinds, positive feedback detector and negative feedback detector. Positive feedback detector's output is higher when the RF signal is larger, and negative feedback detector's output is lower when the RF signal is larger. The feedback circuit can give the feedback to baseband chip, then the output signal from the baseband can be controlled, and RF system's output is controlled to the design target. The design circuit is shown in Fig. 3.

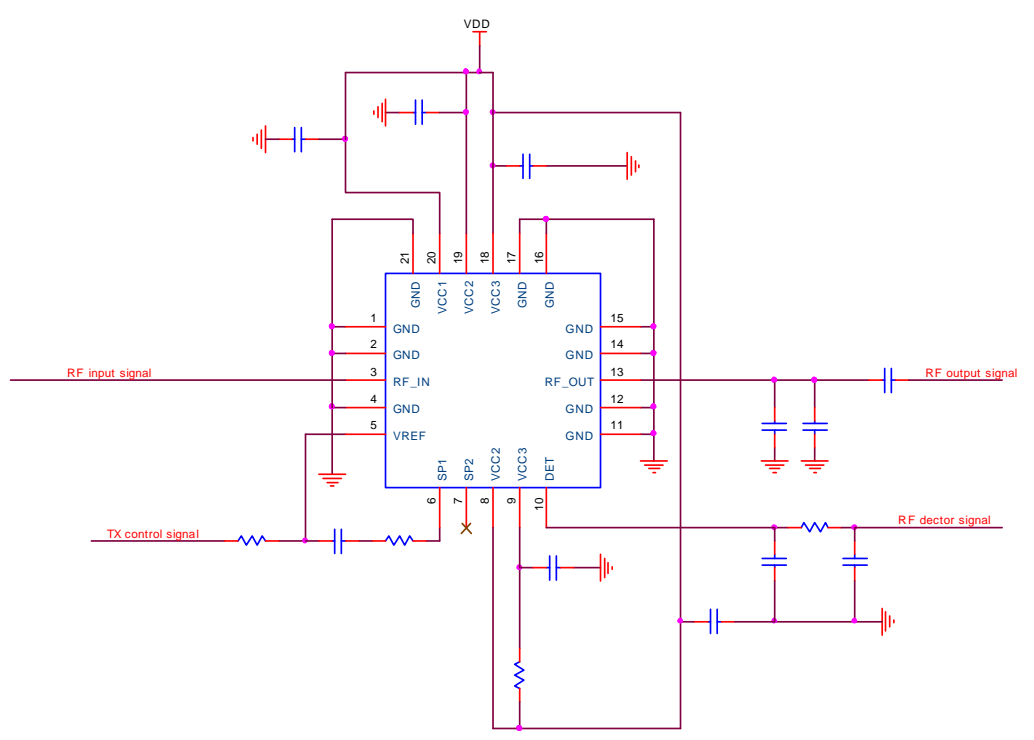

Fig. 3. Power Amplifier Circuit Design

BFP840 is selected to work as RX LNA in this design. Comparing to other common LNA, BFP840 supports up to $6 \mathrm{GHz}$ frenquency band. It not only has gain up to $12 \mathrm{db}$, but also has very small noise figure (the minimum noise figure is $0.6 \mathrm{db}$ ). Paper selects SKY13374 to work as transmitting / receiving switch. Comparing to $2.4 \mathrm{GHz}$ design, Paper add matching capacitor to control signals. This capacitor can filter the harmonic signal to ground and make the TX/RX trace cleaner. This cap's value should be optimized. Paper designs the matching cap by $2.2 \mathrm{pf}$, whose resonant frequency is about $5.5 \mathrm{GHz}$. The design circuit is shown in Fig. 4.

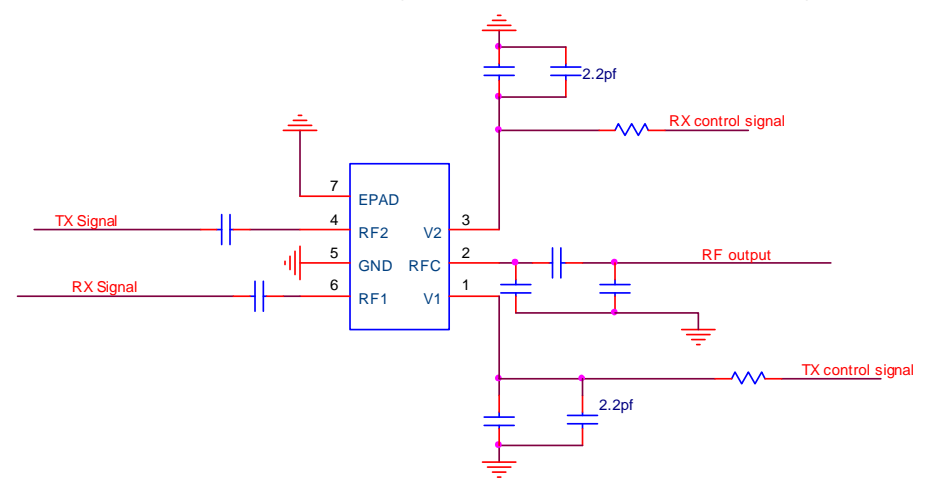

Fig. 4. RF switch design circuit

After finishing the schematic, layout, sample SMT, debugging and calibration on both TX path and $\mathrm{RX}$ path, this design reaches the design target. 


\section{Performance Evaluation}

After commissioning and calibration, the sample can reach the design target, and the output power is up to $24 \mathrm{dbm}$. Wireless network tester Litepoint IQ2010 is used to do radio test in lab.

TX test result. Tested the txpower, spectrum mask, frequency accurancy, EVM, and etc by IQ2010 in lab. When setting the DUT to work at HT20 MCS7 rate, the maximum output power is up to $23.6 \mathrm{dbm}$ and the EVM is $-30.0 \mathrm{db}$. The result is $2 \mathrm{db}$ better than the FCC standard which is $-28 \mathrm{db}$ at this rate. Test result is shown in Fig. 5.

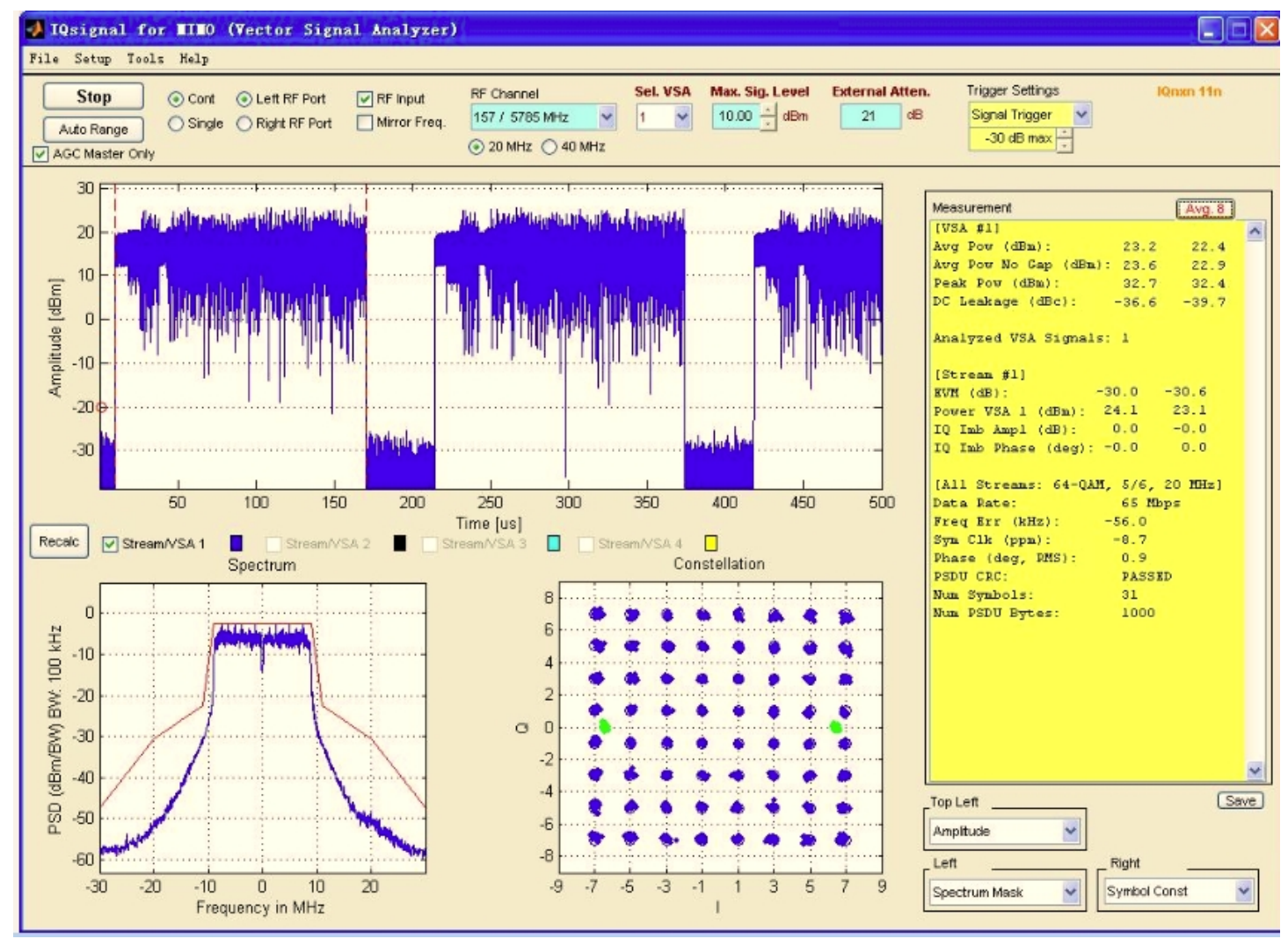

RX test result. Tested the DUT's receiving sensivity in lab, and the RX sensitivity test results are shown in Table 1.

Table 1 . RX sensitivity test results

\begin{tabular}{|l|l|l|l|}
\hline \multicolumn{4}{|l|}{ RX Sen Test result } \\
\hline Freq/MHz & Rate/bps & Chain & $\begin{array}{l}\text { RX } \\
\text { Sen/dbm }\end{array}$ \\
\hline 5180 & $54 \mathrm{M}$ & 0 & -75.9 \\
\hline 5500 & $54 \mathrm{M}$ & 0 & -74.6 \\
\hline 5785 & $54 \mathrm{M}$ & 0 & -73.7 \\
\hline 5180 & $54 \mathrm{M}$ & 1 & -75.9 \\
\hline 5500 & $54 \mathrm{M}$ & 1 & -75.7 \\
\hline 5785 & $54 \mathrm{M}$ & 1 & -72.5 \\
\hline
\end{tabular}

Throughput Test result. Test the board in the working environment by the throughput test tool chariot, we get the test result is up to $170 \mathrm{Mbps}$. 


\section{Conclusions}

This paper designs and implements a $5 \mathrm{GHz}$ mining access point. The test results show that this access point can reach $149.921 \mathrm{Mbps}$ throughput. It can solve the $2.4 \mathrm{GHz}$ wireless systems' signal interference problem, and then provide better hardware support to variety of mine applications, such as video surveillance, wireless communication systems, and etc.

\section{Acknowledgements}

This work was financially supported by the National High Technology Research and Development Program of China (863 Program, Grant 2011AA060406).

\section{References}

[1] Eldad Perahia, Robert Stacey: Next Generation Wireless LANs: Throughput, Robustness, and Reliability in 802.11n. Cambridge University Press (2008)

[2] Barbosa A.V., Caetano, M.F., Barreto P.S., in: IEEE802.11b/g Standard: Theoretical Maximum Throughput[C]. International Conference on Computing, Networking and Communications (ICNC), Page(s): 197 - 201 (2010)

[3] Sarkar, N.I., Lo E., in: Indoor Propagation Measurements for Performance Evaluation of IEEE 802.11 $[$ [C].Telecommunication Networks and Applications Conference (2008): 163 - 168

[4] 802.11n-2009-IEEE Standard for Information technology- Local and metropolitan area networks- Specific requirements- Part 11: Wireless LAN Medium Access Control (MAC)and Physical Layer (PHY) Specifications Amendment 5: Enhancements for Higher Throughput. (2009)

[5] Xiao Lv ${ }^{1, a^{*}}$, Da Zhang, Feng Jin, Xu Liu, Xi Han, in: Research of Mining High Power 802.11n Access Point [C].ICCIPT,2014: 447 - 452 (2014) 